\title{
Highly diversified core promoters in the human genome and their effects on gene expression and disease predisposition
}

\author{
Hemant Gupta ${ }^{1 \dagger}$, Khyati Chandratre ${ }^{1 \dagger}$, Siddharth Sinha ${ }^{1 \dagger}$, Teng Huang ${ }^{1 \dagger}$, Xiaobing $\mathrm{Wu}^{1}$, Jian $\mathrm{Cui}^{2}$,
} Michael Q. Zhang ${ }^{3}$ and San Ming Wang ${ }^{1 *}$ (D)

\begin{abstract}
Background: Core promoter controls transcription initiation. However, little is known for core promoter diversity in the human genome and its relationship with diseases. We hypothesized that as a functional important component in the genome, the core promoter in the human genome could be under evolutionary selection, as reflected by its highly diversification in order to adjust gene expression for better adaptation to the different environment.

Results: Applying the "Exome-based Variant Detection in Core-promoters" method, we analyzed human corepromoter diversity by using the 2682 exome data sets of 25 worldwide human populations sequenced by the 1000 Genome Project. Collectively, we identified 31,996 variants in the core promoter region $(-100$ to +100$)$ of 12,509 human genes (https://dbhcpd.fhs.um.edu.mo). Analyzing the rich variation data identified highly ethnic-specific patterns of core promoter variation between different ethnic populations, the genes with highly variable core promoters, the motifs affected by the variants, and their involved functional pathways. eQTL test revealed that 12\% of core promoter variants can significantly alter gene expression level. Comparison with GWAS data we located 163 variants as the GWAS identified traits associated with multiple diseases, half of these variants can alter gene expression.

Conclusion: Data from our study reals the highly diversified nature of core promoter in the human genome, and highlights that core promoter variation could play important roles not only in gene expression regulation but also in disease predisposition.
\end{abstract}

Keywords: Core promoter, Variation, 1000 genomes, Exome, eQTL, GWAS

\section{Background}

Transcription initiation is the gateway for gene expression. In eukaryotic cells, RNA polymerase II-mediated transcriptional initiation is regulated by the basal transcriptional machinery of cis- and trans-elements in the core promoter region surrounding the transcriptional start site (TSS). The well-known core cis-elements

\footnotetext{
* Correspondence: sanmingwang@um.edu.mo

${ }^{\dagger}$ Hemant Gupta, Khyati Chandratre, Siddharth Sinha and Teng Huang contributed equally to this work.

${ }^{1}$ Cancer Centre and Institute of Translational Medicine, Faculty of Health Sciences, University of Macau, Macau, SAR, China

Full list of author information is available at the end of the article
}

consist of TFIIB recognition element (BRE), TATA box, Initiator element (Inr), downstream promoter element (DPE) etc. and their flanking sequences. The trans-elements of the preinitiation complex (PIC) consist of RNA polymerase II and six general transcription factors TFIIA, TFIIB, TFIID, TFIIE, TFIIF and TFIIH [1-10]. Variation in cis sequences can interfere cis-trans interaction and therefore modulate gene expression in physiological condition; mutation in cis sequences can cause abnormal gene expression contributing to pathological consequences [11-16], such as the mutation in factor IX core promoter in hemophilia B and Hemophilia B

(c) The Author(s). 2020 Open Access This article is licensed under a Creative Commons Attribution 4.0 International License, which permits use, sharing, adaptation, distribution and reproduction in any medium or format, as long as you give appropriate credit to the original author(s) and the source, provide a link to the Creative Commons licence, and indicate if changes were made. The images or other third party material in this article are included in the article's Creative Commons licence, unless indicated otherwise in a credit line to the material. If material is not included in the article's Creative Commons licence and your intended use is not permitted by statutory regulation or exceeds the permitted use, you will need to obtain permission directly from the copyright holder. To view a copy of this licence, visit http://creativecommons.org/licenses/by/4.0/ The Creative Commons Public Domain Dedication waiver (http://creativecommons.org/publicdomain/zero/1.0/) applies to the data made available in this article, unless otherwise stated in a credit line to the data. 
Leyden [17, 18], and the mutation in PKLR promoter in pyruvate kinase (PK)-deficient anemia [19]. Besides, substantial core promoters are lack of the conventional core promoter elements, such as the canonical TATA box, enriched with exceptionally high STR sequences, and islands of purine/pyrimidine [20]. Therefore, studying core promoter cis sequence variation can help to understanding the mechanisms of gene expression regulation and to identifying pathogenic mutations contributing to diseases.

Although the basic features of core promoter have been extensively studied in very details $[1,2]$, genetic diversity in core promoter at the population level remain poorly understood [21]. Extensive efforts made in past decades have collected massive gene expression data under various physiological and pathogenic conditions. However, such data mainly reflect the phenotypic change of gene expression but not the genotypic change in the regulatory machinery, including core promoter. The investigation of altered regulatory machinery, either the cis sequences or the trans factors (e.g. promoters, enhancers, insulators and their binding factors), is vital for revealing the mechanism of normal physiology and disease pathology. Lack of core promoter diversity information is attributed to multiple factors. A key factor is related to the mapping difficulties of core promoter region due to the highly conserved nature of core promoter sequences. To overcome this obstacle, we developed the "Exome-based Variant Detection in Corepromoters (EVDC)" method for core promoter sequence analysis [22]. The principle of this method is to collect core promoter sequences in exome sequences derived from the $5^{\prime}$ UTR probes. This method provides high sensitivity and specificity to map core promoter at genome-level.

In this study, we used our EVDC method to systematically analyse core promoter diversity by using the 2682 exome data sets from 25 human populations collected by the 1000 Genome Project [23]. Our study revealed the highly diversified nature of core promoter sequences in the human genome, tested the effects of core promoter variations in gene expression, and identified the core promoter variants as the GWAS related disease traits.

\section{Results}

\section{Core promoter mapping and variant calling}

The 1000 Genome Project provides rich genome sequence data to study population genetic diversity for specific functional components in the human genome. Using the "Exome-based Variant Detection in Corepromoters" method, we obtained the core promoter sequences from a total of 2640 exome data sets of $25 \mathrm{hu}-$ man ethnic populations collected by the 1000 Genome Project (Supplementary Table 1). Core promoters are classified into two classes, the canonical core promoter that contains typical motifs and major TSS site(s), and the non-canonical core promoter that is lack of typical motifs and with multiple TSS sites [24, 25]. The core promoter region was set TSS $+/-100 \mathrm{bps}$ in order to cover both canonical and non-canonical core promoters within the distance by exome-derived core promoter sequences [22]. We called core promoter variants from the core promoter- mapped sequences. On the condition that a variant should be present in at least two individuals per population, we identified 31,996 distinct variants in 13,586 core promoters of 12,509 genes in the 25 populations, $90.4 \%$ were reported by the 1000 Genome data but $9.4 \%$ per population on average $(4.2-17.5 \%)$ were absent in the 1000 Genome data (Table 1, Supplementary Table 2).

\section{Differences of variant distribution between whole genome and core promoter}

The 1000 Genome study provided quantitative measures for the four types of genomic variants in human populations: 1) Private to population; 2) Private to continent; 3) Shared across continents; and 4) Shared across all continents. We performed a side-by-side comparison of the four types of variants between whole genome and core promoter. We observed that the proportion of each type variants were substantial differences between whole genome variation and core promoter variation in all populations except PUR (Fig. 1, Supplementary Table 3). For instance, in JPT, the ratios in the four types of variants were $15 \%: 48,10 \%: 7,10 \%: 22,65 \%: 23 \%$ between whole genome and core promoter $\left(p<2.8 \mathrm{e}^{237}\right)$, accordingly. While the variants in Africa populations were well preserved in non-Africa populations reflecting human evolution history (Shared across Continent, Shared across all Continents), variants of population-specific (Private to population, Private to continent) increased in nonAfrica population of East Asia and South Asia populations (Fig. 1, Supplementary Table 4). YRI had 55 variants shared in 109 of 110 individuals within the YRI population but only 6 variants as Private to YRI. In CDX, however, 19 of the 27 most common variants were Private to CDX. There were few private variants in America and Europe populations, possibly due to their closer relationship than Asia populations to the Africa ancestors.

\section{Differences of variation between populations}

It is well determined that the African genomes are the most diversified in the humans [23]. The top 7 populations with the highest whole genome variation were ACB, ASW, ESN, GWD, LWK, MSL, and YRI (Fig. 1b in Ref. 231000 Genomes Project Consortium, 2015). However, data shows that this is not always the case in the 
Table 1 Variants called in core promoters in each population ( $>=2$ individuals/population)

\begin{tabular}{|c|c|c|c|c|c|c|c|}
\hline \multirow[t]{2}{*}{ Population } & \multirow[t]{2}{*}{ Variants } & \multicolumn{2}{|c|}{ Classification(\%) } & \multicolumn{3}{|c|}{ Types of variation (\%) } & \multirow{2}{*}{$\begin{array}{l}\text { Ts/ } \\
\text { Tv } \\
\text { ratio }\end{array}$} \\
\hline & & 1000 Genome & Novel & Substitution & Insertion & Deletion & \\
\hline YRI & 11176 & $10441(92.4)$ & $844(7.6)$ & $9682(86.6)$ & $671(6.0)$ & $823(7.4)$ & 3.67 \\
\hline ESN & 7669 & $7046(91.5)$ & $653(8.5)$ & $6634(86.5)$ & $424(5.5)$ & $611(8.0)$ & 3.39 \\
\hline PEL & 7623 & $6251(82.5)$ & $1331(17.5)$ & $6705(88.0)$ & $418(5.5)$ & $500(6.6)$ & 3.41 \\
\hline IBS & 7379 & $6751(91.0)$ & $667(9.0)$ & $6290(85.2)$ & $506(6.9)$ & $583(7.9)$ & 3.61 \\
\hline PUR & 6685 & $6092(91.2)$ & $588(8.8)$ & $5849(87.5)$ & $337(5.0)$ & $500(7.5)$ & 3.77 \\
\hline CEU & 6477 & $5781(89.8)$ & $663(10.2)$ & $5574(86.1)$ & $417(6.4)$ & $486(7.5)$ & 3.80 \\
\hline $\mathrm{GIH}$ & 6387 & $5874(91.2)$ & $559(8.8)$ & $5492(86.0)$ & $426(6.7)$ & $470(7.4)$ & 3.52 \\
\hline $\mathrm{CHB}$ & 6354 & $5688(88.5)$ & $730(11.5)$ & $5312(83.6)$ & $488(7.7)$ & $554(8.7)$ & 3.65 \\
\hline ASW & 5760 & $5286(91.7)$ & $477(8.3)$ & $5053(87.7)$ & $296(5.1)$ & $411(7.1)$ & 3.60 \\
\hline GWD & 5567 & $5081(91.4)$ & $480(8.6)$ & $4807(86.3)$ & $328(5.9)$ & $432(7.8)$ & 3.34 \\
\hline MSL & 5072 & 4834 (95.4) & $231(4.6)$ & 4531 (89.3) & $237(4.7)$ & $304(6.0)$ & 3.67 \\
\hline PJL & 4561 & 3999 (87.1) & 589 (12.9) & 3816 (83.7) & $317(7.0)$ & $428(9.4)$ & 3.50 \\
\hline ITU & 4402 & 3907 (88.9) & $490(11.1)$ & 3798 (86.3) & $351(8.0)$ & $253(5.7)$ & 3.33 \\
\hline$A C B$ & 4308 & 3984 (95.0) & $216(5.0)$ & 3806 (88.3) & $212(4.9)$ & $290(6.7)$ & 3.90 \\
\hline STU & 4282 & 3786 (87.8) & $523(12.2)$ & 3607 (84.2) & $304(7.1)$ & 371 (8.7) & 3.55 \\
\hline LWK & 4245 & 4047 (95.8) & $178(4.2)$ & 3839 (90.4) & 153 (3.6) & $253(6.0)$ & 3.94 \\
\hline BEB & 3746 & 3228 (89.2) & $404(10.8)$ & 3332 (88.9) & $190(5.1)$ & $224(6.0)$ & 3.78 \\
\hline TSI & 3617 & $3223(89.0)$ & 399 (11.0) & $3104(85.8)$ & 215 (5.9) & $298(8.2)$ & 3.85 \\
\hline CLM & 3016 & $2728(92.0)$ & $240(8.0)$ & $2659(88.2)$ & $160(5.3)$ & $197(6.5)$ & 3.58 \\
\hline $\mathrm{CHS}$ & 2995 & 2637 (87.7) & 369 (12.3) & $2526(84.3)$ & $216(7.2)$ & $253(8.4)$ & 3.28 \\
\hline CDX & 2948 & $2576(86.7)$ & 391 (13.3) & $2515(85.3)$ & 189 (6.4) & $244(8.3)$ & 3.25 \\
\hline FIN & 1803 & 1646 (91.6) & $151(8.4)$ & $1586(88.0)$ & $81(4.5)$ & $136(7.5)$ & 3.94 \\
\hline GBR & 1315 & $1213(91.1)$ & $117(8.9)$ & $1121(85.2)$ & $77(5.9)$ & 117 (8.9) & 3.87 \\
\hline JPT & 953 & $879(92.2)$ & $74(7.8)$ & $814(85.4)$ & $59(6.2)$ & $80(8.4)$ & 3.81 \\
\hline KHV & 747 & $650(87.3)$ & $95(12.7)$ & $643(86.1)$ & $45(6.0)$ & $59(7.9)$ & 3.37 \\
\hline Ave. total & 4763 & 4305 (90.4) & 457 (9.6) & $643(86.1)$ & $284(6.0)$ & $355(7.5)$ & 3.60 \\
\hline
\end{tabular}

core promoter region. Of the top seven populations with the highest core promoter variation (YRI, ESN, PEL, IBS, PUR, CEU and GIH), only two African populations of YRI and ESN were included (Fig. 2a). The differences imply that positive selection was stronger in core promoter than in the whole genome in reflecting the importance of altered gene expression regulation in nonAfrica populations for better adaptation of their new environments after moving out of Africa. The actual numbers of variants and their affected core promoters were also substantially different between different populations as reflected by nearly 15 -fold more variation in YRI than in KHV, in which YRI had 11,176 variants in 6542 core promoters whereas KHV had only 747 variants in 646 core promoters (Table 1, Fig. 2a).

\section{Type of variants, repetitive sequences and Ts/Tv ratio}

Simple repeats are well determined to be enriched in core promoter region under evolutionary selection to regulate gene expression, and can be pathogenic as genetic predisposition for psychiatric diseases including schizophrenia [26], bipolar disorder [27]. Studies suggested that repetitive sequences can be enriched in regulatory region to play roles in gene expression regulation $[25,28,29]$. On average, $13.5 \%$ variants were insertion or deletion (Table 1). We analyzed the insertions and deletions from all the 25 populations using RepeatMasker program and determined that nearly all were simple repeats but no classical repetitive sequences of $A l u, B 1$, and LINE etc. (Supplementary Table 5). Lack of these types of repetitive sequences suggest that repetitive sequences do not play significant roles in altering core promoter sequences to influence gene expression. Tv/Ts ratio have been extensively tested in exonic, intronic, intergenic, miRNA, lncRNA and whole genome but not in core promoter region [30-32]. Our calculation shows that the $\mathrm{Ts} / \mathrm{Tv}$ ratio in core promoter variants were at 3.60 per population on average (3.25 to 3.90 ) in the 25 


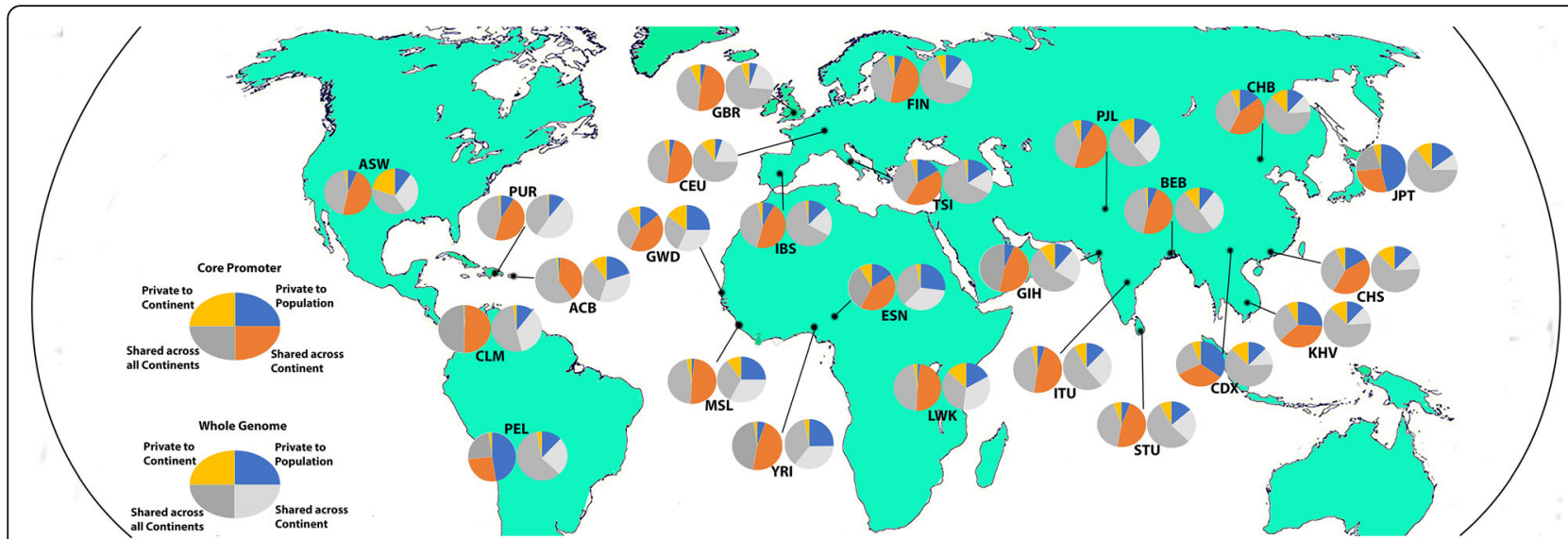

Fig. 1 Comparison of genome variation and core promoter variation in worldwide human populations. The variants in each population are divided into four groups, including Shared across all continents, Shared across the continent, Private to continent, and Private to population. The distribution of variants is compared in pair between core promoter and whole genome. In each pair, the left circle is from core promoter variants and the right circle is from whole genome referred from Fig. 1a (1000 Genomes Project Consortium, 2015). See actual rates and $p$ values in Supplementary Table 3). The original world map was downloaded from Free world maps (http://www.free-world-maps.com). The figure was generated by using Adobe Photoshop version CS6

populations (Table 1). The rates were much higher than the 3 in the coding region and 2.0-2.1 across the genome.

\section{Variation upstream and downstream of TSS}

The variation upstream TSS was lower than the variation downstream TSS (Fig. 2b). This feature may reflect conservation requirement in the upstream TFBSs, whereas downstream is the $5^{\prime}$ UTR region of coding genes, where more mutations and multiple TSSs could introduce more variation. We further compared the distribution of simple repeats. Of the 201 STRs distributed in the core promoter region, 159 were upstream but only 41 were downstream. This distribution is consistent with the previous observation [33]. Possible reason could be that long STRs in the 5'UTR region could affect on mRNA stability therefore less tolerated at the downstream.

\section{Functional categories for the genes with highly variable core promoters}

A group of genes had high variation frequencies in their core promoters. For example, the core promoter of PRSS1 had 26 variants, distributing 137 times in 19 populations (Fig. 2c, Supplementary Fig. 1). Interestingly PRSS1 5'UTR SNPs are known to be associated with chronic pancreatitis [34]. Using geneSCF tool, we searched 4172 genes with $>=10$ core promoter variants per core promoter in the REACTOME, a database for biological pathway classification [35] (https://reactome. org). The most frequent pathway was signal transduction (492 genes). Others included metabolism, immune system, signaling by $\mathrm{G}$ protein-coupled receptors (GPCR), and post-translational protein modification (Table 2, Supplementary Table 6).

\section{Variants in core promoter motifs}

A total of 970 variants were located at the core promoter motifs of BRE, TATA box, Inr and DPE for 3306 times (Fig. 3). Of these four motifs, TATA box had the lowest number of variants of one per population on average, indicating that TATA box doesn't tolerate variation as its base composition is critical for TBP (TATA-binding protein) binding to open double-strand DNA for transcription initiation. Of other three motif sequences, Inr and BRE had modest degree of variation but DPE had the highest number of variants throughout all 25 populations. This indicates that DPE is a favorable motif for variation selection to influence transcription initiation. The lower variation in TATA box, Inr and BRE is consistent with lower degree of variation upstream of TSS.

\section{Core-promoter variants and expression change}

The variant in core promoter is the genotype. We use Expression Quantitative Trait Loci (eQTL) to test if the genotype variation can have phenotype change as reflected by altered expression in the variant-located gene. With expression data from 53 genotyped human tissue types of 10,688 samples, the eQTL database provides a useful tool for comprehensive test of the association of core promoter variation on gene expression (https://gtexportal.org/home/tissueSummaryPage). We analyzed the entire 31,996 core promoter variants, of which 23,608 variants were identified from the 1000 Genome data, we identified 3814 core promoter variants $(12 \%)$ that can cause altered gene expression $(p<0.05)$ 


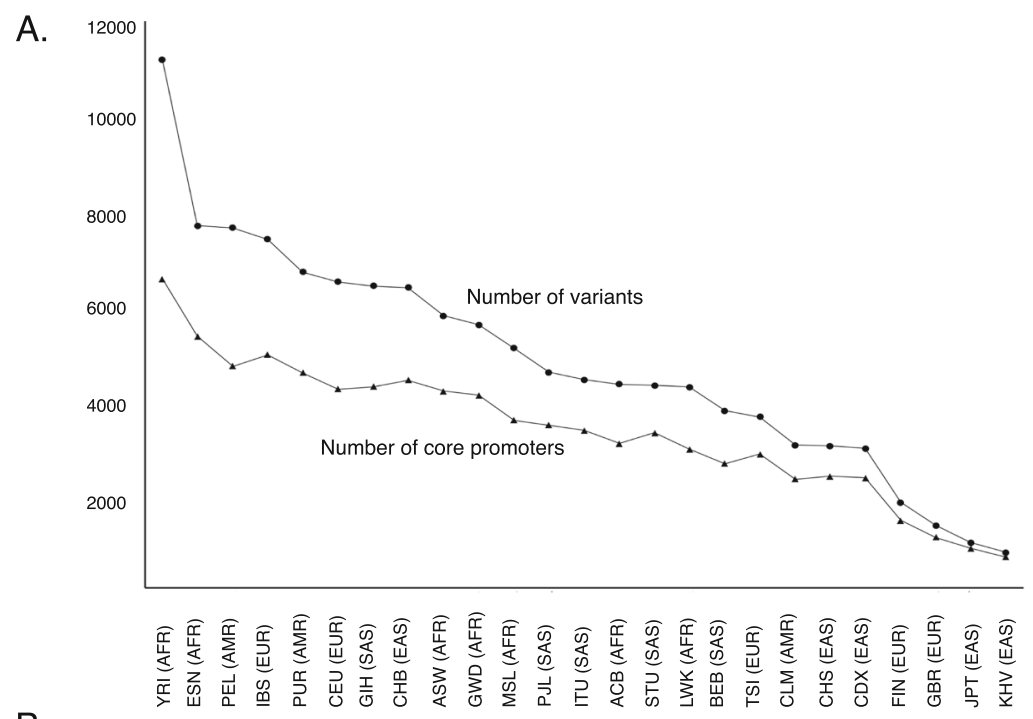

B.

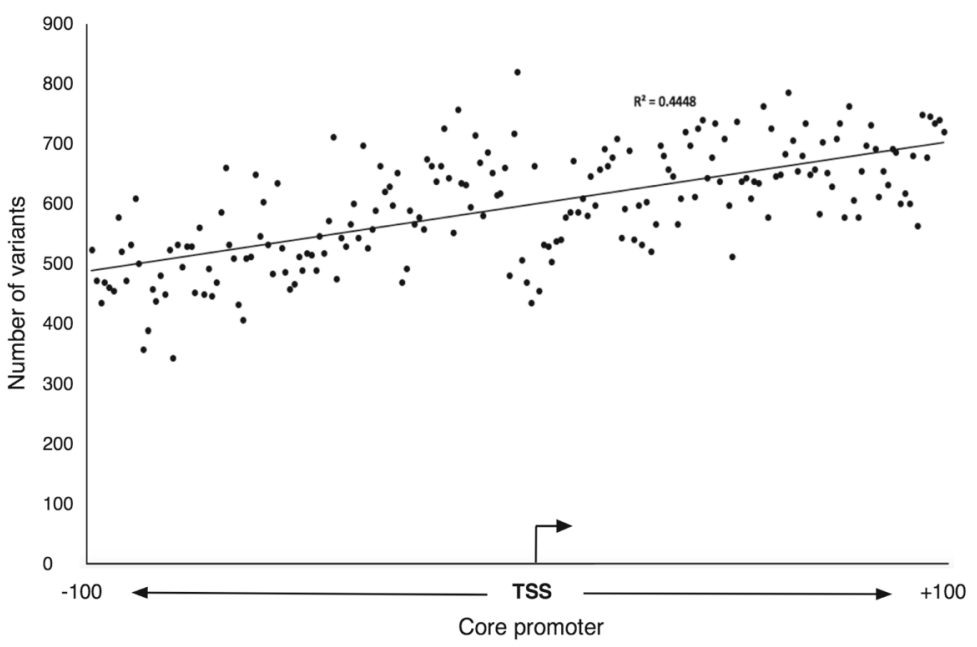

C.

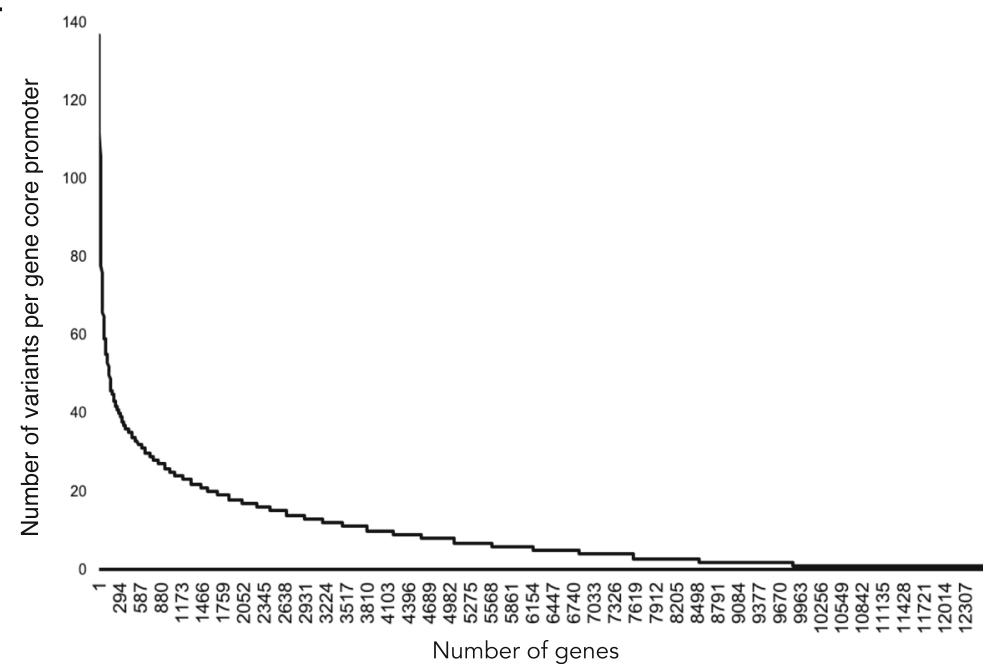

Fig. 2 (See legend on next page.) 
(See figure on previous page.)

Fig. 2 Features of core promoter variation in human populations. a core promoter variants and their hosting genes across human populations. It shows the highly diversified core promoter among human populations, with YRI having the highest variation frequency whereas KHV the lowest. b. Variant distribution across core promoter region. It shows that variation downstream of TSS site was higher than the variation upstream TSS. $\mathbf{c}$. Variation frequencies in the core promoters of 12,509 genes containing core promoter variants

from at least one out the 53 tissue types (Supplementary Table 7). Taking DNA damage repair genes as the example: There are 177 genes within this functional category (supplementary Table 8A). Among these genes, 94 (52\%) including MSH2, MSH3, PMS2, RAD51C, RECQL5, $X P C$ and $X R C C 2$ had variants in their core promoters. eQTL test showed that all the variants in the 94 genes can cause expression changes (Fig. 4a, supplementary Table $8 \mathrm{~B})$. For example, core promoter of $M S H 3$, the gene involved in post-replicative DNA mismatch repair [36], had 6 variants, distributed in 53 individuals in 19 populations (Fig. 4b). Of the 6 variants, 5 variants could decrease

Table 2 Functional classes for genes with highly variable core promoters

\begin{tabular}{ll}
\hline Functional pathways & Number of genes \\
\hline Signalling & 115 \\
Cytokine Signaling in Immune system & 225 \\
G alpha (s) signalling events & 301 \\
GPCR downstream signalling & 201 \\
Olfactory Signaling Pathway & 492 \\
Signal Transduction & 307 \\
Signaling by G protein-coupled receptors (GPCR) \\
Metablism \\
Metabolism \\
Metabolism of proteins \\
Metabolism of RNA & 395 \\
Immune & 397 \\
Adaptive Immune System & 172 \\
Immune System & 110 \\
Innate Immune System & 328 \\
Gene expression & 189 \\
Gene expression (Transcription) & \\
Generic Transcription Pathway & 231 \\
RNA Polymerase II Transcription & 170 \\
Others & 232 \\
Cell Cycle & 113 \\
Developmental Biology & $4695^{*}$ \\
Disease & \\
Post-translational protein modification & \\
Total* & \\
\hline
\end{tabular}

*Including genes involved in multiple functional pathways
MHS3 expression whereas one (rs1105525 at +42$)$ might increase its expression (Fig. 4c).

\section{Core promoter variants as disease predispositions}

Extensive GWAS studies have identified a large number of genetic traits associated with many human diseases, the majority are located in non-coding regions. The rich core promoter variants across diverse populations provide a resource to test if any GWAS identified disease trait loci could be located in core promoter region. GWAS catalog (the variant depository database) contains 55,297 trait loci associated with 2887 diseases or phenotypes identified from 3541 studies [37] (accessed on $8 / 28 / 2018$ ). Searching the 31,996 core promoter variants in GWAS Catalog identified 163 (0.5\%) with matched GWAS traits $(0.29 \%$ of total 55,297 GWAS Catalog collection), associated with 163 diseases or phenotypes (5.6\% of the 2887 GWAS analyzed diseases or phenotypes) (Supplementary Table 9A, 9B, 9C, 9D, 9E, 9F). Most of the diseases or phenotypes matched by core promoter variants/GWAS traits were present in multiple ethnic populations reflecting the common variant / common disease model of GWAS design. However, a few population-specific variants did exist, such as the variants associated with asthma present only in Africa, body mass index only in South Asia, prostate cancer (advanced) only in East Asia, dementia with Lewy bodies only in South Asia, and bone density only in Europe. Such variants suggest the presence of population-specific traits for the associated diseases or phenotypes (Fig. 5a), an issue in interpreting GWAS results [38]. Further eQTL test in the 163 variants revealed that 83 (50.9\%) variants might affect the expression of the target genes (Supplementary Table 10). For examples, rs1883832 is a variant associated with susceptibility to chronic hepatitis B infection in Chinese population [39]. It is located in CD40 core promoter at -90 (Fig. $5 \mathrm{~b}$ ). eQTL test showed that this variant could significantly increase CD40 expression in multiple tissue types, with lung as the most affected (Fig. 5c). Another example is for the germline mutations in BRCA1 and BRCA2, in which germline mutation increases risk of hereditary breast cancer. As substantial cases maintain intact coding sequences, alteration of regulatory region was considered as a potential mechanism for the missing heritability [40]. The fact that only 5 variants were present in $B R C A 1$ core promoter in few populations and none were in $B R C A 2$ core 


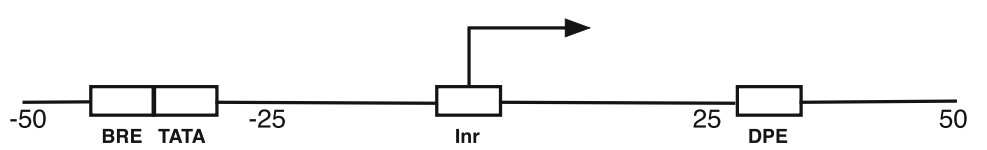

\begin{tabular}{|c|c|c|c|c|c|c|c|}
\hline \multirow[t]{2}{*}{ Population } & \multirow[t]{2}{*}{ Tota variants } & \multicolumn{4}{|c|}{ Motif } & \multirow[t]{2}{*}{ Total } & \multirow[t]{2}{*}{ Rate in motif } \\
\hline & & BRE & TATA & INR & DPE & & \\
\hline YRI & 11,176 & 33 & 3 & 46 & 211 & 293 & 0.026 \\
\hline PEL & 7,623 & 21 & 1 & 41 & 158 & 221 & 0.029 \\
\hline ESN & 7,669 & 33 & 2 & 43 & 137 & 215 & 0.028 \\
\hline IBS & 7,379 & 23 & 1 & 37 & 144 & 205 & 0.028 \\
\hline $\mathrm{GIH}$ & 6,387 & 17 & 1 & 32 & 134 & 184 & 0.029 \\
\hline PUR & 6,685 & 13 & 2 & 38 & 125 & 178 & 0.027 \\
\hline CEU & 6,477 & 18 & 0 & 31 & 127 & 176 & 0.027 \\
\hline $\mathrm{CHB}$ & 6,354 & 16 & 1 & 35 & 118 & 170 & 0.027 \\
\hline GWD & 5,567 & 18 & 3 & 29 & 113 & 163 & 0.029 \\
\hline ASW & 5,760 & 12 & 2 & 29 & 101 & 144 & 0.025 \\
\hline PJL & 4,561 & 17 & 1 & 22 & 93 & 133 & 0.029 \\
\hline MSL & 5,072 & 9 & 1 & 33 & 89 & 132 & 0.026 \\
\hline ITU & 4,402 & 17 & 1 & 19 & 90 & 127 & 0.029 \\
\hline STU & 4,282 & 11 & 0 & 23 & 86 & 120 & 0.028 \\
\hline $\mathrm{ACB}$ & 4,308 & 6 & 3 & 26 & 75 & 110 & 0.026 \\
\hline BEB & 3,746 & 12 & 1 & 24 & 72 & 109 & 0.029 \\
\hline LWK & 4,245 & 6 & 1 & 23 & 78 & 108 & 0.025 \\
\hline TSI & 3,617 & 9 & 1 & 24 & 69 & 103 & 0.028 \\
\hline CLM & 3,016 & 7 & 1 & 16 & 76 & 100 & 0.033 \\
\hline $\operatorname{cDx}$ & 2,948 & 16 & 0 & 19 & 61 & 96 & 0.033 \\
\hline $\mathrm{CHS}$ & 2,995 & 5 & 1 & 15 & 64 & 85 & 0.028 \\
\hline FIN & 1,803 & 7 & 0 & 12 & 40 & 59 & 0.033 \\
\hline GBR & 1,315 & 2 & 1 & 6 & 27 & 36 & 0.027 \\
\hline JPT & 953 & 1 & 0 & 6 & 15 & 22 & 0.023 \\
\hline $\mathrm{KHV}$ & 747 & 2 & 0 & 2 & 13 & 17 & 0.023 \\
\hline Average & & 13 & 1 & 25 & 93 & 132 & 0.028 \\
\hline Total & & 331 & 28 & 631 & 2,316 & 3,306 & \\
\hline
\end{tabular}

Fig. 3 Variants distributed in the four core promoter motifs. It shows that DPE had the highest number of variants and TATA box had the lowest across all 25 populations

promoter excludes core promoter as the mutationtargeted region.

By mining the exome data from 25 human ethnic populations, our study provides deep insight for core promoter diversity in the human genome, highlighting that core promoter variation can play more important roles than thought in gene expression regulation and diseases. An immediate use of the core promoter variation information is that it provides ethnic-based references to study core promoter variation in diseases, which can be more precise than the non-ethnic-based variation references in distinguishing between disease-causing mutation and ethnic-specific core promoter variation.

\section{Discussion}

Although core-promoter occupies only a small part of the regulatory region, it is the gateway in controlling onoff of transcription through cis-trans interaction between core-promoter motifs and transcriptional initiation complex composed of various transcriptional factors. As the cis-trans interaction within the tight space needs to be extremely precise, a single base change in core promoter sequences could have profound impact on transcription.
Our mapping analysis in 25 worldwide human ethnic populations reveals the highly diversified nature of human core-promoter sequences as reflected by the differential presence of core promoter variants between ethnic human populations. We identified the genes with highly variable core promoters, observed the effects of core promoter variation in altering gene expression, identified the functional pathways affected by the core promoter variation, and located multiple core promoter variants as the traits associated with multiple diseases identified GWAS.

The patterns of core-promoter variation distribution were substantially different from the ones of genomewide variation distribution. As shown in Fig. 1, in all 24 populations except PUR (Puerto Ricans) population, the differences of variant distribution between genome and core promoter were highly significant in all four sections of "Private to population", Private to continent", "Shared across continent" and "Shared across all continents". For example, the differences in YRI were $4.25 \mathrm{xE}^{-74}$. The differential variation distribution likely reflects the consequences of evolution selection in the core promoters to fine-turn gene expression for better fitness of the ethnic 
A.

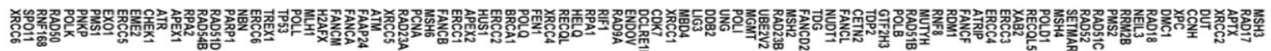

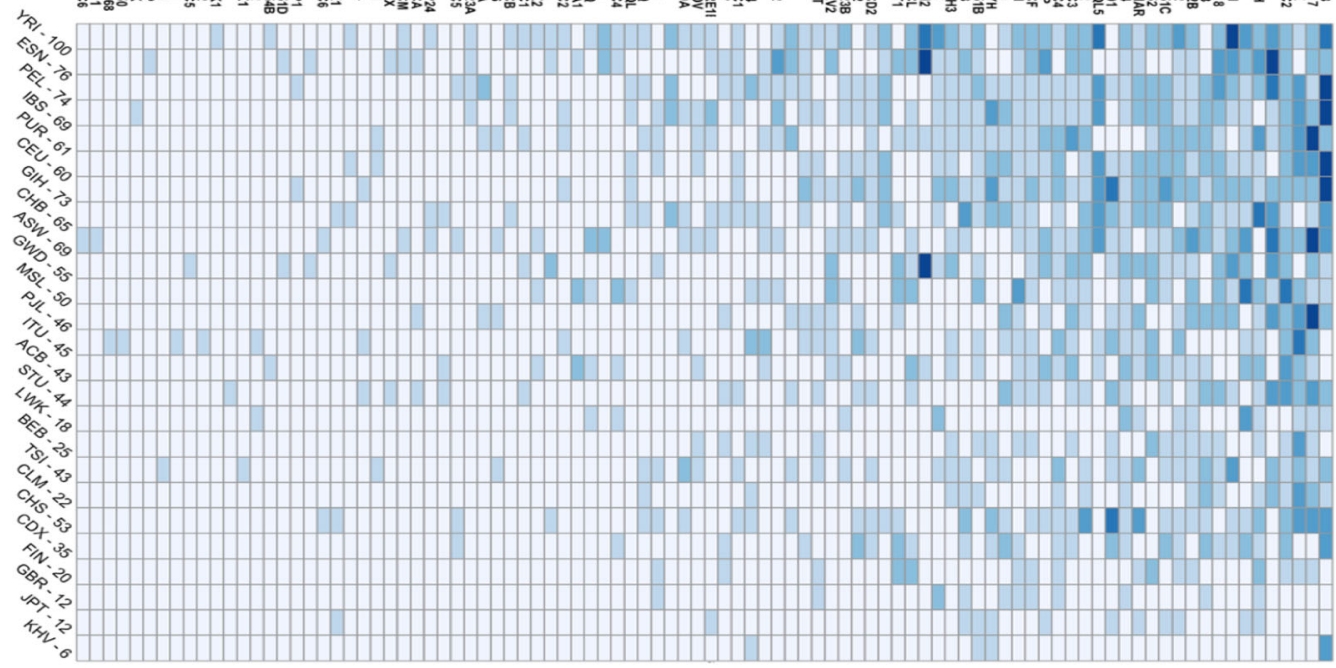

B.

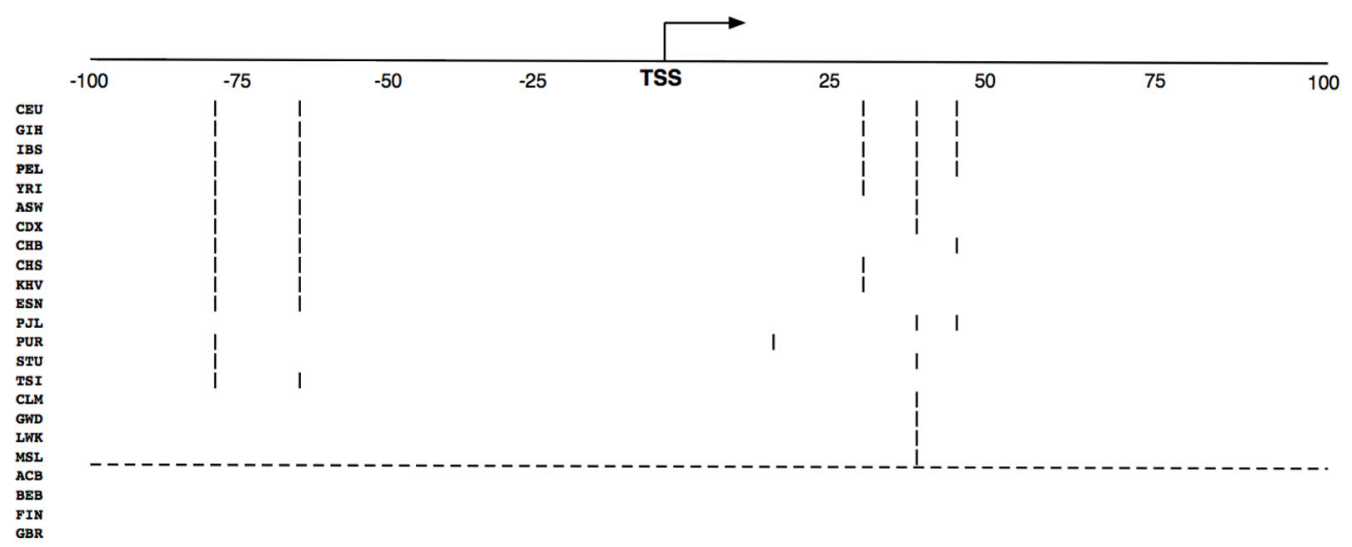

c.

Thyroid eQTL 5_79950389_GC_G_b37 ENSG00000113318.9

Cells_Transformed_fibroblasts eQTL 5_79950508_C_T_b37 ENSG0000011331
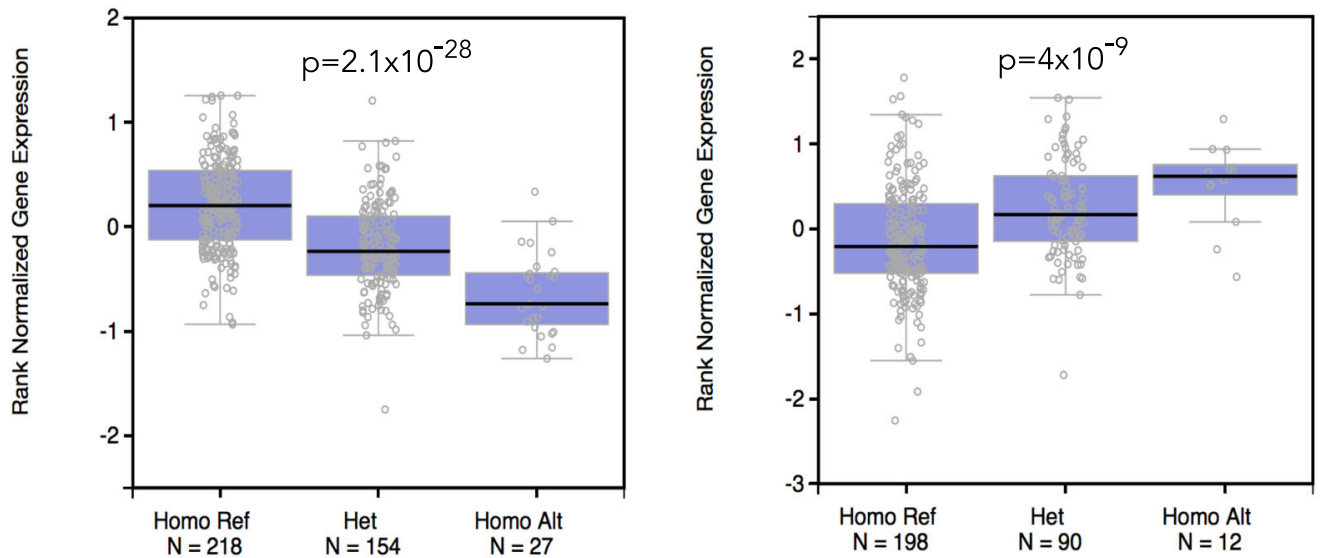

Fig. 4 (See legend on next page.) 
(See figure on previous page.)

Fig. 4 Core promoter variation in DNA damage repair genes and their effects in gene expression. a. Variant distribution in core promoters of DNA damage repair genes. Of the 177 genes involved with DNA damage repair, 94 genes had variants in their core promoters with the highest in YRI and the lowest in KHV. b. Variants in MSH3 core promoter. Six variants were distributed 53 times in MSH3 core promoter in 19 populations, of which 5 variants were present in 18 populations. $\mathbf{c}$. Variants in MSH3 core promoter alter MSH3 expression. eQTL test showed that the variant rs 151182735 at -76 can decrease MSH3 expression level in thyroid $\left(p=2.1 \times 10^{-28}\right)$, and the variant rs 1105525 at +42 can increase MSH3 expression level in transformed fibroblast cells $\left(p=4.0 \times 10^{-9}\right)$

population to their specific environments. Furthermore, there were substantial differences of core promoter variation between different ethnic populations as exemplified by the 15 -fold more core promoter variants in YRI population than in KHV population. This may suggest that certain natural environments were much friendly for the survival of resident populations that there was not much stress to select the expression-regulatory machinery whereas other natural environments were much harsh for the resident populations that heavy selection on expression-regulatory machinery might have occurred for better adaptation of the resident populations. Among the pathways affected by core promoter variation, signal transduction pathway was affected the most, indicating the importance of signal transduction in connecting between environment stimulation and gene response for better fitness.

The relationship between core promoter variation and pathogenic consequences is particularly interesting. While core promoter variation plays important roles in adaptation, certain variation could pass the threshold of homeostasis leading to abnormal phenotypes. Most of the disease-associated traits identified by GWAS are located in intergenic region. A set of core promoter variants coincident with the disease traits identified by GWAS provides direct evidence for the involvement of core promoter mutations associated with diseases, and provides a bridge to connect GWAS-identified disease traits for functional study of their roles in disease development.

Decoding the biological basis of genetic variation in any part of the genome can use bottom-up approach of using phylogenetic, genetic diversity, segregation, and biostatistics, etc. The top-bottom approach of harvesting the "lower-handling fruits" can also be applied. In our study, we addressed the issue of core promoter diversity by using the 1000 Genome data, the first human genome data derived from representative ethnic populations across the global. Continuous efforts are needed to reach comprehensive understanding of genetic basis of core promoter variation in human population and the roles of core promoter variation in human diseases.

\section{Conclusions}

Our comprehensive study reveals the highly diversified nature of the core promoter in human populations, and demonstrates more important roles than thoughts of core promoter variation in gene expression regulation and disease predisposition.

\section{Methods}

\section{Exome data sources}

The exome data generated by the 1000 Genome Project was used in the study [23] (ftp://ftp.1000genomes.ebi.ac. $\mathrm{uk} / \mathrm{vol} 1 / \mathrm{ftp})$. The data contain 2640 exome data sets from 25 different ethnic populations except Mexican Ancestry from Los Angeles USA (MXL) population (Supplementary Table 1, http://www.internationalgenome. org/data-portal/sample).

\section{Ethic statement}

The data used by the study included these from public databases including 1000 Genome Project database (ftp://ftp.1000genomes.ebi.ac.uk/vol1/ftp), GTEx database (https://gtexportal.org/home/), and GWAS database (https://www.ebi.ac.uk/gwas/). Therefore, ethical approval is not required for this study.

\section{Mapping core promoter sequence and calling variants} Burrows-Wheeler Aligner (BWA version 0.7.15) using mem algorithm was used to map exome sequences to the human reference genome sequences (hg19). The resulting SAM (Sequence Alignment/Map) files were converted into BAM files and sorted using Samtools utility (version 1.3.1). Duplicates were removed using MarkDuplicates and the read group information was added using AddOrReplaceReadGroups, functions of Picard tools (version 1.119), respectively. The BAM files were further processed using Genome Analysis Toolkit (GATK version 3.7) with its recommended best practices pipeline for variant calling and GATK BaseRecalibrator was used for base quality score recalibration. GATK HaplotypeCaller was used for variant calling (includes InDel realignment) in gVCF mode, followed by joint genotyping with GenotypeGVCFs, variant recalibration with VariantRecalibrator and filtration of low-quality genotypes with VariantFiltration. The called variants were further annotated using ANNOVAR for gene-based (RefSeq genes), region-based (cytoband, genomicSuperDups) and filter-based annotation (1000G, dbSNP147, ExAC, cosmic70 and ClinVar). Minimum depth for calling variants was set to 10 . Core promoter sequences from the 
A.

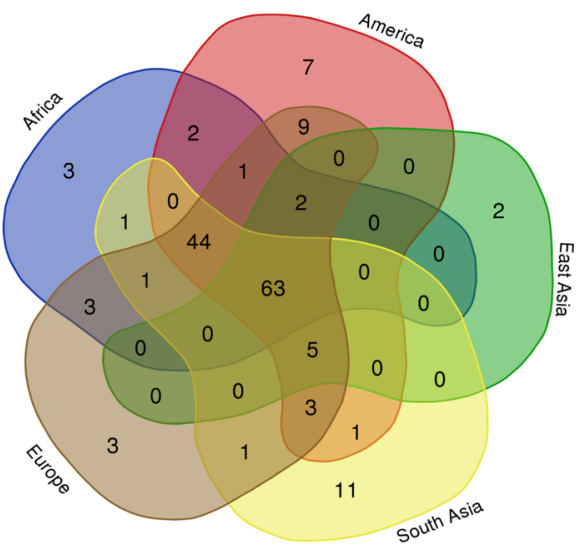

B.

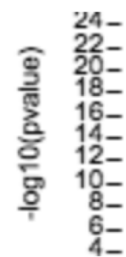

$\circ$ 。
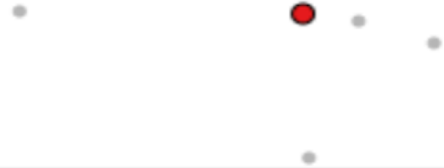

GWAS Catalog | |

Genes

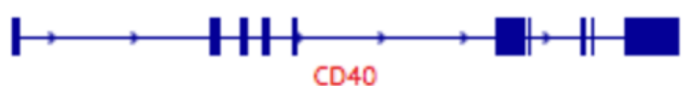

C.

rs1883832/CD40/Lung

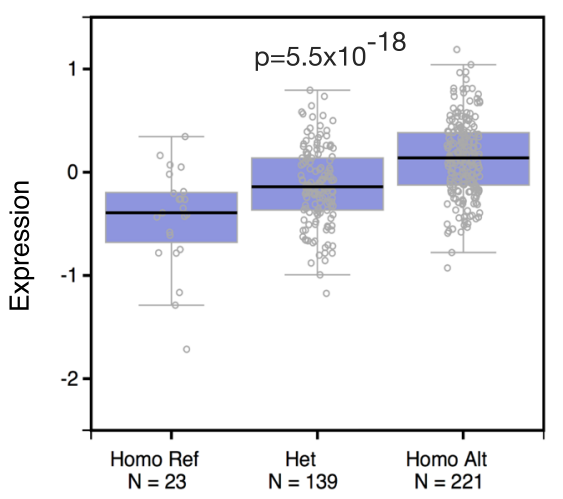

Fig. 5 Core promoter variants associated with diseases. a. Venn demonstration of multiple diseases identified by core promoter variants matched with GWAS traits in different populations. Of the 163 diseases or phenotypes, 31 were shared in populations across all continents, 44 were shared in populations across the continents of Africa, American, Europe, and South Asia but not in East Asia. The rests were private to population or private to continental. b. Variant rs 1883832 in CD40 core promoter. CD40 is a member of the TNF-receptor superfamily involving in immune and inflammatory responses. A variant rs 1883832 is located at the core promoter of CD40 (+ 90). This variant was determined by GWAS study as being associated with high susceptibility to chronic hepatitis B infection in Chinese population. c. eQTL analysis showed rs1883832 can increase CD40 expression in lung $\left(p=5.5 \times 10^{-18}\right)$ 
reference genome hg19 were extracted and used as the reference to identify the core promoter region as defined TSS (transcription start site) +/- 100 bps. The following consensus sequences were used for motif analysis: BRE (SSRC GCC), TATA box (TATAWAAR), INR (YYANWYY), and DPE (RGWYV). S: C/G; R:A/G; W:A/C; Y:C/T; N:A/C/G/ $\mathrm{T} ; \mathrm{V}: \mathrm{A} / \mathrm{C} / \mathrm{G}$ (IUPAC notation, International Union of Pure and Applied Chemistry) The called variants were compared to 1000 Genome, dbSNP147, and ExAC databases to distinguish between the known variants and novel variants. Novel variants were deposited in dbSNP (Supplementary Table 11). An open access database, dbHuman CorePromoter Variation, hosting human core-promoter variation was developed for public exploration of core promoter variation data (https://dbhcpd.fhs.um.edu.mo).

eQTL analysis and GWAS-disease associated trait analysis For eQTL analysis, core promoter variants were searched in Genotype-Tissue Expression database [41, 42] (GTEx, https:/gtexportal.org/home/) to locate their position and to determine their potential influence on gene expression. Of the mapped variants, those with the $p$-value $<0.05$ were used as the representative for causing expression changes in the affected tissue type. For GWAS-disease trait analysis, core promoter variants were searched in the GWAS Catalog (https://www.ebi.ac.uk/gwas/). The identified variants were tested in GTEx for their potential effects on gene expression in human tissues. The functional categories of variant-affected genes were classified by searching the REACTOME database using geneSCF tool [43].

\section{Statistics analysis}

Chi-squared test was used to test the distribution differences between whole genome variation and core promoter variation. The analysis was performed using SPSS 19.0.

\section{Availability of data and materials}

All data analyzed in this study are included in this published article, its supplementary information files and database.

\section{Supplementary information}

The online version contains supplementary material available at https://doi. org/10.1186/s12864-020-07222-5.

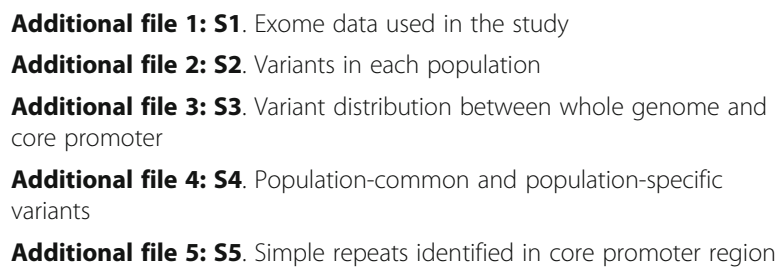

Additional file 6: S6. Functional classification of genes with highfrequent core promoter variation

Additional file 7: S7. Effects of core promoter variants on gene expression by $\mathrm{eQTL}$ analysis

Additional file 8: S8. Core promoter variation in DNA damage repair genes

Additional file 9: S9. Disease-associated core promoter variants Additional file 10: S10. Effects of core promoter-located GWAS-traits on gene expression by eQTL analysis

Additional file 11: S11. Novel variants identified from the study Additional file 12: S Figure 1. PRSS1 core promoter variation

\section{Abbreviations}

eQTL: Expression quantitative trait Loci; GWAS: Genome wide association study; UTR: Untranslated region; EVDC: Exome-based Variant Detection in Core-promoters; TSS: Transcriptional start site; GTEx: Genotype-tissue expression database

\section{Acknowledgements}

We are thankful to the Information and Communication Technology Office (ICTO), University of Macau for providing the High-Performance Computing Cluster (HPCC) resource and facilities for the study.

\section{Authors' contributions}

HG, KC, SS, XW: data curation and analysis; TH: database construction; CJ: statistics data analysis; MQZ: design, data interpretation; SMW: funding, conceptualization, design, data interpretation, and manuscript writing. All authors have read and approved the manuscript.

\section{Funding}

This work was supported by grants from the Macau Science and Technology Development Fund (085/2017/A2), Macau Science and Technology Development-Ministry of Science and Technology of People's Republic of China fund (0077/2019/ AMJ), the University of Macau (SRG2017-00097-FHS, MYRG2019-00018-FHS), and an innovation grant (FHSIG/SW/0007/2020P) and a startup fund from the Faculty of Health Sciences, University of Macau to SMW. The funders played no roles in the design of the study, in the collection, analysis and interpretation of data and in writing the manuscript.

\section{Availability of data and materials}

The datasets supporting the conclusions of this article are available in the URL https://dbhcpd.fhs.um.edu.mo and Supplementary Tables 1, 2, 3, 4, 5, 6, 7, 8, 9, 10, 11 and Supplementary Fig. 1. The novel variants identified from the study have been deposited in NCBI dbSNP database (Supplementary Table 11).

Ethics approval and consent to participate

Not applicable.

\section{Consent for publication}

Not applicable.

\section{Competing interests}

All authors declare no competing interests.

\section{Author details}

${ }^{1}$ Cancer Centre and Institute of Translational Medicine, Faculty of Health Sciences, University of Macau, Macau, SAR, China. ${ }^{2}$ Eppley Institute for Cancer Research, University of Nebraska Medical Center, Omaha, NE 68198, USA. ${ }^{3}$ Department of Biological Sciences, Center for Systems Biology, University of Texas at Dallas, Richardson, TX 75080, USA.

Received: 19 May 2020 Accepted: 9 November 2020

Published online: 30 November 2020

References

1. Smale T, Kadonaga T. The RNA polymerase II core promoter. Ann Rev Biochem. 2003;72:449-79.

2. Kadonaga JT. Perspectives on the RNA polymerase II core promoter. Wiley Interdiscip Rev Dev Biol. 2012;1:40-51. 
3. Vo Ngoc L, Wang YL, Kassavetis GA, Kadonaga JT. The punctilious RNA polymerase II core promoter. Genes Dev. 2017;31:1289-301.

4. Batut P, Dobin A, Plessy C, Carninci P, Gingeras TR. High-fidelity promoter profiling reveals widespread alternative promoter usage and transposondriven developmental gene expression. Genome Res. 2012;23:169-80.

5. Roy AL, Singer DS. Core promoters in transcription: old problem, new insights. Trends Biochem Sci. 2015;40:165-71.

6. Zhao B, Cao JF, Hu GJ, Chen ZW, Wang LY, Shangguan XX, Wang LJ, Mao YB, Zhang TZ, Wendel JF, et al. Core cis-element variation confers subgenome-biased expression of a transcription factor that functions in cotton fiber elongation. New Phytol. 2018;218:1061-75.

7. Watanabe K, Kokubo T. SAGA mediates transcription from the TATA-like element independently of Taf1p/TFIID but dependent on core promoter structures in Saccharomyces cerevisiae. PLoS One. 2017;12:e0188435.

8. Sato MP, Makino T, Kawata M. Natural selection in a population of Drosophila melanogaster explained by changes in gene expression caused by sequence variation in core promoter regions. BMC Evol Biol. 2016;16:35.

9. Lubliner S, Regev I, Lotan-Pompan M, Edelheit S, Weinberger A, Segal E. Core promoter sequence in yeast is a major determinant of expression level. Genome Res. 2015;25:1008-17.

10. Srivastava R, Rai KM, Srivastava M, Kumar V, Pandey B, Singh SP, Bad SK, Singh BD, Tuli R, Sawant SV. Distinct role of core promoter architecture in regulation of light-mediated responses in plant genes. Mol Plant. 2014;7: 626-41

11. Wray GA. The evolutionary significance of cis-regulatory mutations. Nat Rev Genet. 2007:8:206-16.

12. Albert FW, Kruglyak $\mathrm{L}$. The role of regulatory variation in complex traits and disease. Nat Rev Genet. 2015;16:197-212.

13. Poulos RC, Thoms JA, Shah A, Beck D, Pimanda JE, Wong JW. Systematic screening of promoter regions pinpoints functional cis-regulatory mutations in a cutaneous melanoma genome. Mol Cancer Res. 2015;13:1218-26.

14. Lappalainen T, Montgomery SB, Nica AC, Dermitzakis ET. Epistatic selection between coding and regulatory variation in human evolution and disease. Am J Hum Genet. 2011:89:459-63.

15. Sharma A, Jiang C, De S. Dissecting the sources of gene expression variation in a pan-cancer analysis identifies novel regulatory mutations. Nucleic Acids Res. 2018;46:4370-81.

16. Gurdasani D, Carstensen T, Tekola-Ayele F, Pagani L, Tachmazidou L, Hatzikotolas K, Karthikeyan S, lles L, Pollard MO, Choudhury A, et al. The African Genome Variation Project shapes medical genetics in Africa. Nature. 2015;517:327-32.

17. Crossley M, Brownlee GG. Disruption of a C/EBP binding site in the factor IX promoter is associated with haemophilia B. Nature (London). 1990;345:4446.

18. Reijnen MJ, Sladek FM, Bertina RM, Reitsma PH. Disruption of a binding site for hepatocyte nuclear factor 4 results in hemophilia B Leyden. Proc Natl Acad Sci U S A. 1992;89:6300-3.

19. Manco L, Ribeiro ML, Máximo V, Almeida H, Costa A, Preitas O, Barbot J, Abade A, Tamagnini G. A new PKLR gene mutation in the R-type promoter region affects the gene transcription causing pyruvate kinase deficiency. $\mathrm{Br}$ J Haematol. 2000;110:993-7.

20. Darvish H, Nabi MO, Firouzabadi SG, Karimlou M, Heidari A, Najmabadi H, Ohadi M. Exceptional human core promoter nucleotide compositions. Gene. 2011:475:79-86.

21. Horn S, Figl A, Rachakonda PS, Fischer C, Sucker A, Gast A, Kadel S, Moll I, Nagore E, Hemminki K, et al. TERT promoter mutations in familial and sporadic melanoma. Science. 2013;339:959-61.

22. Kim YC, Cui J, Luo J, Xiao F, Downs B, Wang SM. Exome-based Variant Detection in Core Promoters. Sci Rep. 2016:6:30716.

23. 1000 Genomes Project Consortium, Auton A, Brooks LD, Durbin RM, Garrison EP, Kang HM, Korbel JO, Marchini JL, McCarthy S, McVean GA, et al. A global reference for human genetic variation. Nature. 2015;526:68-74.

24. FANTOM Consortium and the RIKEN PMI and CLST (DGT), Forrest AR, Kawaji H, Rehli M, Baillie JK, de Hoon MJ, Haberle V, Lassmann T, Kulakovskiy IV, Lizio M, et al. A promoter-level mammalian expression atlas. Nature. 2014; 507(7493):462-70.

25. Sawaya S, Bagshaw A, Buschiazzo E, Kumar P, Chowdhur S, Black MA, Gemmell N. Microsatellite tandem repeats are abundant in human promoters and are associated with regulatory elements. PLoS One. 2013;8: e54710
26. Emamalizadeh B, Movafagh A, Darvish $H$, Kazeminasab S, Andarva M, Namdar-Aligoodarzi P, Ohadi M. The human RIT2 core promoter short tandem repeat predominant allele is species-specific in length: a selective advantage for human evolution? Mol Gen Genomics. 2017;292:611-7.

27. Alizadeh F, Moharrami T, Mousavi N, Yazarlou F, Bozorgmehr A, Shahsavand E, Delbari A, Ohadi M. Disease-only alleles at the extreme ends of the human ZMYM3 exceptionally long 5' UTR short tandem repeat in bipolar disorder: A pilot study. J Affect Disord. 2019;251:86-90.

28. Bolton KA, Ross JP, Grice DM, Bowden NA, Holliday EG, Avery-Kiejda KA, Scott RJ. STaRRRT: a table of short tandem repeats in regulatory regions of the human genome. BMC Genomics. 2013;14:795.

29. Weber $\mathrm{LL}$, Wong C. Mutation of human short tandem repeats. Hum Mol Genet. 1993;2:1123-8.

30. Bainbridge MN, Wang M, Wu Y, Newsham I, Muzny DM, Jefferies JL, Albert TJ, Burgess DL, Gibbs RA, et al. Targeted enrichment beyond the consensus coding DNA sequence exome reveals exons with higher variant densities. Genome Biol. 2011;12:R68.

31. Wang J, Raskin L, Samuels DC, Shyr Y, Guo Y. Genome measures used for quality control are dependent on gene function and ancestry. Bioinformatics. 2015;31:318-23.

32. International HapMap Consortium. The International HapMap Project. Nature. 2003:426(6968):789-96.

33. Namdar-Aligoodarzi P, Mohammadparast S, Zaker-Kandjani B, Talebi Kakroodi S, Jafari Vesiehsari M, Ohadi M. Exceptionally long 5' UTR short tandem repeats specifically linked to primates. Gene. 2015;569:88-94.

34. Hezel AF, Kimmelman AC, Stanger BZ, Bardeesy N, Depinho RA. Genetics and biology of pancreatic ductal adenocarcinoma. Genes Dev. 2006;20: 1218-49.

35. Jassal B, Matthews L, Viteri G, Gong C, Lorente P, Fabregat A, Sidiropoulos K, Cook J, Gillespie M, Haw R, et al. The reactome pathway knowledgebase. Nucleic Acids Res. 2020;48(D1):D498-503.

36. Song H, Ramus SJ, Quaye L, DiCioccio RA, Tyrer J, Lomas E, Shadforth D, Hogdall E, Hogdall C, McGuire V, et al. Common variants in mismatch repair genes and risk of invasive ovarian cancer. Carcinogenesis. 2006;27:2235-42.

37. Morales J, Welter D, Bowler EH, Cerezo M, Harris LW, McMahon AC, Hall P, Junkins HA, Milano A, Hastings E, et al. A standardized framework for representation of ancestry data in genomics studies, with application to the NHGRI-EBI GWAS Catalog. Genome Biol. 2018;19:21.

38. Martin AR, Gignoux CR, Walters RK, Wojcik GL, Neale BM, Gravel S, Daly MJ, Bustamante CD, Kenny EE. Human Demographic History Impacts Genetic Risk Prediction across Diverse Populations. Am J Hum Genet. 2017;100:63549.

39. Jiang DK, Ma XP, Yu H, Cao G, Ding DL, Chen H, Huang HX, Gao YZ, Wu XP, Long XD, et al. Genetic variants in five novel loci including CFB and CD40 predispose to chronic hepatitis B. Hepatology. 2015;62:118-28.

40. Downs B, Wang SM. Epigenetic changes in BRCA1-mutated familial breast cancer. Cancer Genet. 2015;208:237-40.

41. Clarke L, Fairley S, Zheng-Bradley X, Streeter I, Perry E, Lowy E, Tassé AM, Flicek P. The international Genome sample resource (IGSR): A worldwide collection of genome variation incorporating the 1000 Genomes Project data. Nucleic Acids Res. 2017;45:D854-9.

42. Gamazon ER, Segrè AV, van de Bunt $M$, Wen $X, X i H S$, Hormozdiari F, Ongen $H$, Konkashbaev A, Derks EM, Aguet F, et al. Using an atlas of gene regulation across 44 human tissues to inform complex disease- and traitassociated variation. Nat Genet. 2018:50:956-67.

43. Subhash S, Kanduri C. GeneSCF: a real-time based functional enrichment tool with support for multiple organisms. BMC Bioinformatics. 2016:17:365.

\section{Publisher's Note}

Springer Nature remains neutral with regard to jurisdictional claims in published maps and institutional affiliations. 